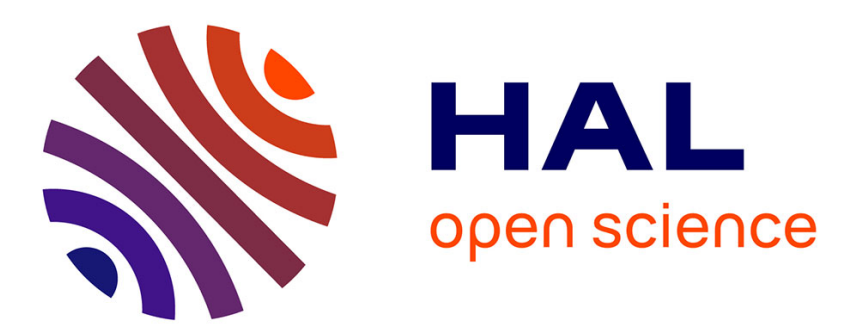

\title{
Control of Adhesion using Surface Functionalisations for Robotic Microhandling.
}

Jérôme Dejeu, Patrick Rougeot, Sophie Lakard, Michaël Gauthier

\section{To cite this version:}

Jérôme Dejeu, Patrick Rougeot, Sophie Lakard, Michaël Gauthier. Control of Adhesion using Surface Functionalisations for Robotic Microhandling.. IEEE/RSJ International Conference on Intelligent Robots and Systems, IROS'12., Oct 2012, Vilamoura, Algarve, Portugal. pp.2307-2312. hal-00772424

\section{HAL Id: hal-00772424 https://hal.science/hal-00772424}

Submitted on 10 Jan 2013

HAL is a multi-disciplinary open access archive for the deposit and dissemination of scientific research documents, whether they are published or not. The documents may come from teaching and research institutions in France or abroad, or from public or private research centers.
L'archive ouverte pluridisciplinaire HAL, est destinée au dépôt et à la diffusion de documents scientifiques de niveau recherche, publiés ou non, émanant des établissements d'enseignement et de recherche français ou étrangers, des laboratoires publics ou privés. 


\title{
Control of Adhesion using Surface Functionalisations for Robotic Microhandling
}

\author{
Jérôme Dejeu ${ }^{1}$, Patrick Rougeot ${ }^{1}$, Sophie Lakard $^{2}$ and Michaël Gauthier ${ }^{1}$, IEEE Member
}

\begin{abstract}
Robotic microhandling is a promising way to assemble microcomponents in order to manufacture new generation of Hybrid Micro ElectroMechanical Systems (HMEMS). However, at the scale of several micrometers, adhesion phenomenon highly perturbs the micro-objects release and the positioning. This phenomenon is directly linked to both the object and the gripper surface mechanical and chemical properties. The control of the adhesion properties requires multidisciplinary approaches including roughness control, mechanical properties control and chemical surface functionalisation. We propose to control adhesion by using chemical surface functionalisations by intrinsic conducting polymer electrodeposition or Self-Assembly Monolayer (SAM) and using surface structuration.
\end{abstract}

\section{INTRODUCTION}

Robotic manipulation in microscale and nanoscale is drastically modified by the well known scale effects [1-3]. Some of the physical effects negligible in the macroscale become predominant in microscale or nanoscale. Concerning the robotic handling of micro-objects the most important problem reported in the literature is the adhesion between the gripper and the object which disturbs its release [2]. Three general approaches are currently used in the literature: (i) to use adhesion as a gripping principle [4,5]; (ii) to overcome adhesion with a stronger effect [6-8] or (iii) to avoid adhesion by using non-contact manipulation [9-11]. The both first approaches require to have a repeatable high level or low level adhesion, respectively. The repeatability of the adhesion between a gripper and a manipulated object is thus a challenge for using or overcoming adhesion in robotic microhandling.

The adhesion is a function of the mechanical and chemical properties of the object's surface. The control of the adhesion properties is required multidisciplinary approaches including roughness control, mechanical properties control and chemical surface functionalisation. The impact of the surrounding

This work was also supported by the EU under FAB2ASM (contract FoF-NMP-2010-260079): Efficient and Precise 3D Integration of Heterogeneous Microsystems from Fabrication to Assembly, and by the FrancheComté Region under FIMICAP (contract 2011C-07333): Fiabilisation du microassemblage et mise au point de capteurs intelligents par ingénierie des surfaces. The force measurement was taken by Atomic Force Microscopy on the NANOROL platform http://nanorol.cnrs.fr/events.php. We would also like to acknowledge the support of Mikhael Bechelany for the surface structuration.

${ }^{1}$ Jérôme Dejeu, Patrick Rougeot, Michaël Gauthier are with FEMTOST Institute, AS2M Department, UMR CNRS 6174 - UFC / ENSMM / UTBM, 24 rue Alain Savary, 25000 Besançon, France firstname.lastname at femto-st.fr

${ }^{2}$ Sophie Lakard is with the UTINAM Institute, CNRS-UMR 6213, Franche-Comté University, 16 route de Gray, 25030 Besançon Cedex, France sophie.lakard at univ-fcomte.fr media which is able to interact with the surface should also be taken into consideration.

The next section deals with an overview of three proposed methods to control adhesion and deals with the device used to measure forces. Each method including polymer functionalisation, nanostructuration and chemical grafting is presented in the following sections.

\section{SURFACE MODIFICATION AND CHARACTERIZATION}

\section{A. General principles}

The modification of the surface properties of both object and gripper can be obtained by different methods. The most used methods are the polyelectrolyte adsorption, the polymer electrodeposition, the molecules grafting (covalent bond between the substrate and the molecules) on the surface. In micro-assembly, the majority of objects are in silicon so the more easy is to graft silane or to electrodeposite polymer. On silicon, it is also easy to control the roughness of the substrate by struturation of the surface by sphere. The different methods have been tested (see in figure 1):

- the electrodeposition of Intrinsic Conducting Polymers (ICP) such as pyrrole (Figure 1a), aniline (Figure 1b), p-phenylenediamine (Figure 1c) and 3,4ethylenedioxythiophene (Figure 1d)

- the surface structuration by PS spheres lithography

- the 3 (ethoxydimethylsilyl) propyl amine (APTES) for the silane grafting (Figure 1e);<smiles>c1cc[nH]c1</smiles>

(a)<smiles>Nc1ccccc1</smiles>

(b)<smiles>Nc1ccc(N)cc1</smiles>

(c)<smiles>c1scc2c1OCCO2</smiles>

(d)

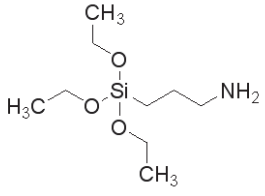

(e)
Fig. 1. Molecules used for the surface functionalisation: a) pyrrole, b) aniline, c) p-phenylenediamine, d) 3,4-ethylenedioxythiophene, e) 3 (ethoxydimethylsilyl) propyl amine.

The polymer electrodeposition is interesting because this ability to localise the film on the surfaces. Indeed, the polymer is deposited only on microelectrodes used during electrodeposition and doesn't cancel the conductivity of the surface. This process enables to pattern polymers on the surfaces of grippers and/or objects. The technique used in order to struturate the surface by the PS spheres is the 
combination of both bottom-up and top-down approaches. With this method, the nanospheres lithography has received great consideration as a result of its simplicity compare to conventional lithography techniques. Using this methods, patterning of a wide variety of solid substrates has been achieved including metals [12], semiconductors [13], and ceramics. The advantage of the nanostructuration is double: an increasing and a control of the roughness. The last approach consists in using chemical functions which can be controlled actively. The protonation (absorption of protons) of particular chemical functions can be controlled in a liquid using the $\mathrm{pH}$.

\section{B. Force measurement}

Force measurements were performed in order to characterize the functionalisations. Force-distance curves were realized using a stand-alone SMENA scanning probe microscope (NT-MDT). The force measurement accomplished on this Atomic Force Microscope (AFM) is based on the measurement of the deformation of the AFM cantilever with a laser deflection sensor. The silicon rectangular AFM cantilever, whose stiffness is near $0.3 \mathrm{~N} / \mathrm{m}$, was fixed and the substrate moved vertically. The force calibration was operated for each cantilever with its resonance frequency, and a first measurement on hard material. As the applicative objective of this work is to improve reliability of microobject manipulation, interactions have been studied between a micrometric sphere and a plane. Measurements were in fact performed with a cantilever where a borosilicate sphere was glued. The majority of the experiments was achieved with a radius $r_{1}$ of the borosilicate sphere on $5 \mu \mathrm{m} .10$ measurements were done at different locations on the same sample with a driving speed of $200 \mathrm{~nm} / \mathrm{s}$. The uncertainly on all pull-off forces values was less than $10 \%$.

\section{FUNCTIONALISATION BY POLYMER ELECTRODEPOSITION}

The first functionalisation consists in the electrodeposition of conductive polymers on surfaces. Different Intrinsic Conducting Polymers (ICP) have been tested in order to decrease the adhesion force between the modified surface and a non-functionalised micrometric sphere.

The intrinsic conductive polymer adsorption was performed by the electropolymerisation on silica or gold. The electropolymerization conditions used in this work to electrosynthesize 3,4-polyethylenedioxythiophene (Pedot), polypyrrole (Ppy), polyaniline (Pani) and poly(pphenylenediamine) (Ppd) have already been detailed by Lupu et al. [14], [15] and by Dejeu et al. [16], [17]. The measurement was done in dry medium just after the film formation (see Figure 2). The point 0 on the distance axis corresponds to the contact point between the cantilever and the surface.

From Table I, it can be deduced that the interactions and adhesion between the cantilever and the surface were strongly modified due to surface functionalisation. Indeed, the adhesion force between the cantilever and the polymerfree substrates was considerable since the adhesion force value was $-1000 \mathrm{nN}$ and $-900 \mathrm{nN}$ for silicon and gold substrates, respectively. These adhesion forces were strongly reduced thanks to electrodeposited polymer films since they varied from $-86.1 \mathrm{nN}$ for Pedot-modified $\mathrm{Au}$ surfaces to $-15.0 \mathrm{nN}$ for Pedot-Si surfaces.

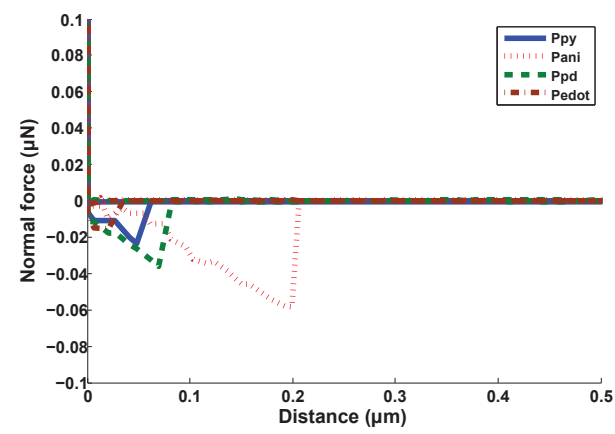

Fig. 2. Adhesion force measurement for the different intrinsic conductivity polymers on silicon: Ppy (blue line), Pani (- - red), Ppd (- - green), Pedot (- - brown).

If force measurements demonstrated the influence of the functionalization on adhesion, they also proved the influence of the substrate, even coated by a polymer film. Indeed, Pani and Ppd films induce higher pull-off forces on Si substrate $(-58.0 \mathrm{nN}$ and $-61.5 \mathrm{nN}$, respectively) than on $\mathrm{Au}$ substrate $(-52.0 \mathrm{nN}$ and $-44.0 \mathrm{nN}$, respectively). On the contrary, Ppy and Pedot induce lower pull-off forces on Si substrate $(-25.0 \mathrm{nN}$ and $-15.0 \mathrm{nN}$, respectively) than on $\mathrm{Au}$ substrate (-62.0 $\mathrm{nN}$ and $-86.1 \mathrm{nN}$, respectively). The most important difference, observed for Pedot films, can be easily explained by the strong affinity of sulfur atoms for gold substrates that has already been extensively studied and used, especially for the elaboration of Self-Assembled Monolayers [18], [19].

Consequently, the handling of micro-objects could be performed by gripper coated by intrinsic conducting polymers, which lead to a drastic decrease of the adhesion between chemically-modified surfaces and micrometric sphere. Moreover, the pull-off force can be varied by choosing the appropriate electrodeposited polymer. Their morphological features must be also analysed for an use in micromanipulation (Figure 3).

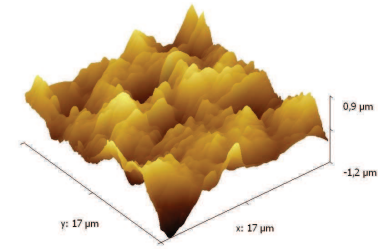

(a)

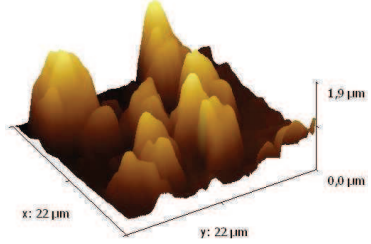

(b)
Fig. 3. Morphologies of the intrinsic conductivity polymers deposit on silicon: a) Pani and b) Ppd. 
TABLE I

PULl-OFF FORCES (NN) MEASURED ON SILICON AND GOLD ELECTRODES FOR DIFFERENT ICPS (CANTILEVER STIFFNESS 0.3 N/M).

\begin{tabular}{cccccc}
\hline \multirow{2}{*}{$\begin{array}{c}\text { Electrode } \\
\text { nature }\end{array}$} & Free of polymer & \multicolumn{4}{c}{ ICPs } \\
\cline { 3 - 6 } & & PPY & PANI & PPPD & PEDOT \\
\hline silicon & $<-1000 \mathrm{nN}$ & $-25 \pm 20 \mathrm{nN}$ & $-58 \pm 20 \mathrm{nN}$ & $-61.5 \pm 30 \mathrm{nN}$ & $-15 \pm 5 \mathrm{nN}$ \\
\hline gold & $<-900 \mathrm{nN}$ & $-62 \pm 20 \mathrm{nN}$ & $-52 \pm 20 \mathrm{nN}$ & $-44 \pm 10 \mathrm{nN}$ & $-86.1 \pm 30 \mathrm{nN}$ \\
\hline
\end{tabular}

From AFM pictures of the different polymers, it can be noticed that all the electrodeposited polymers are not suitable for use in micromanipulation. Indeed, the peak to peak height and width must be smaller than the size of the object, otherwise the object could be blocked between the peaks, and the release could be difficult. In Figure 3, the first film morphologies (Figure 3a) are adapted for the micromanipulation whatever the object size is, whereas the second (Figure 3b) is inappropriate for the object size less than 10 $\mu \mathrm{m}$. The other films have to be improved in order to decrease the roughness. Such control of the morphological features might be performed by optimization of the electrodeposition parameters.

\section{CONTROL OF THE ROUGHNESS BY NANOSTRUCTURATION}

The second approach is based on the control of the roughness of the surface in order to reduce adhesion. In order to obtain a repeatable roughness, the surface is structured with nanospheres placed by self-assembly.

The deposition parameters by spin-coating have been already detailed in previous paper [20]. The spheres were selfassembled into a closed pack (Figure 4a), and after Reactive Ion Etching process (RIE), the radius has decreasing and a non-closed PS spheres has obtained (Figure 4b).

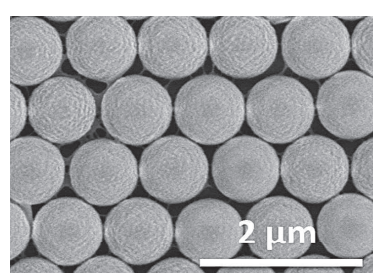

(a)

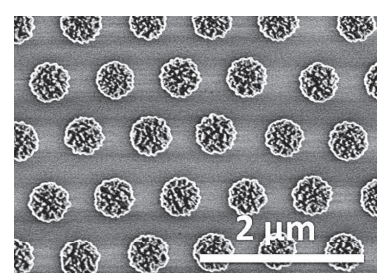

(b)
Fig. 4. Closed (a) and non-closed (b) PS sphere packed deposited on the silica surface.

The experimental measurement can be compared to the adhesion modeling taken into account the sum of the van der Waals forces between a sphere glued into the probe extremity and spheres of the structured surface. In an application case and also during force measurements the location of the sphere on the cantilever up to the structured surface cannot be controlled precisely. When the bead on the cantilever $r_{1}$ is approaching, it touches the nanospheres $r_{2}$ on a noncontrolled position $(\mathrm{i}, \mathrm{j})$. We have shown that the force, function of the Hamaker constant $A_{12}$, is included between a minimum and a maximum which verify [20]:

$$
\begin{aligned}
& F_{\text {min }}=\sum_{i, j}^{\mathbb{Z}^{2}} \frac{A_{12} r_{1} r_{2}}{6 z_{i j}^{2}\left(r_{1}+r_{2}\right)} \cdot \frac{r_{2}+z_{0}+r_{1}}{r_{2}+z_{i j_{\text {min }}}+r_{1}} \\
& F_{\text {max }}=\sum_{i, j}^{\mathbb{Z}^{2}} \frac{A_{12} r_{1} r_{2}}{6 Z_{i j}^{2}\left(r_{1}+r_{2}\right)} \cdot \frac{\sqrt{\left(r_{2}+z_{0}+r_{1}\right)^{2}-\left(4 / 3 \cdot r_{2}^{2}\right)}}{r_{2}+Z_{i j_{\max }}+r_{1}}
\end{aligned}
$$

where:

$$
\begin{aligned}
& z_{i j}=\sqrt{\left(r_{2}+z_{0}+r_{1}\right)^{2}+4 r_{2}^{2}\left(j^{2}-i j+i^{2}\right)}-r_{1}-r_{2} \\
& Z_{i j}=\sqrt{\left(r_{2}+z_{0}+r_{1}\right)^{2}+4 r_{2}^{2}\left(j^{2}-i j-i-j+i^{2}\right)}-r_{1}-r_{2} .
\end{aligned}
$$

The comparison between value predicted by the model and the measurement, plotted in Figure 5, shows a good concordance. Indeed $90 \%$ of the experimental points validate the model. The other $10 \%$ of the experimental points are very near to the predicted value, just few nanoNewtons below the model.

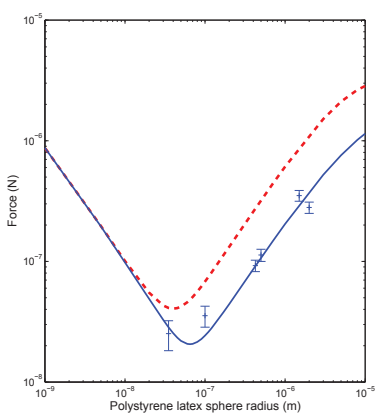

Fig. 5. Comparison between the model (minimum force, blue line, and maximum force, - red) and experimental measurements (error bar) on the structured surface for borosilicate $r_{1}=10 \mu \mathrm{m}$ in function of the nanostructuration $r_{2}$.

The second results deals with the determination of a minimum of the interaction force which represents an optimum of adhesion reduction in the applicative field of micromanipulation. In our experimental case, the optimum radius $r_{2}$ in order to minimize the adhesion is between 45 and $100 \mathrm{~nm}$. If the radius $r_{2}$ is greater than this optimum only 1 or 3 PS sphere(s) induces significant forces and if it is lower, more and more PS spheres should be considered in the sum thus increasing the force. This value depends on the borosilicate sphere radius glued to the cantilever. The model can be extended to different radii of sphere $r_{1}$ using (1) and (2).

The non-closed PS spheres packed surface was obtained from the $900 \mathrm{~nm}$ initial radius PS spheres. So, in the sums (1) and (2) respectively 1 and 3 nanospheres induces 
significant forces. But during the RIE process to decrease the spheres size, plasma bombardment causes roughness on the PS sphere surface [21]. The roughness, or the asperity, due to the etching process can be simulated by nanospheres on the PS spheres periphery with a radius $r_{3}$. Four interaction cases between the probe and the PS spheres are possible (Figure 6):

- Case 1 : The probe is aligned and in contact with one asperity on one PS sphere, Figure 6a

- Case 2 : The probe is in the middle three asperities on one PS sphere and in contact with them, Figure 6b

- Case 3 : The probe is in the middle on the three PS spheres and in contact with one asperity on each PS sphere, Figure 6c

- Case 4: The probe is in the middle on the three PS spheres and in contact with three asperities on each PS sphere, Figure 6d.

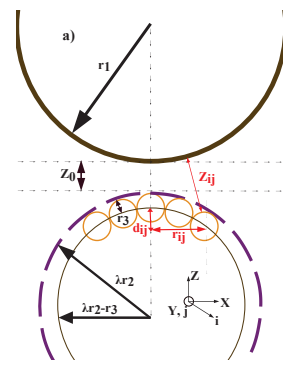

(a)

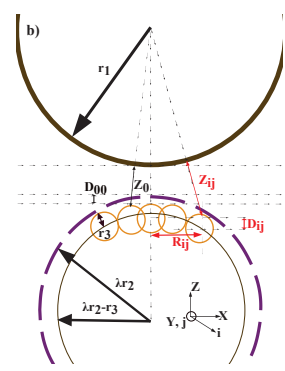

(b)

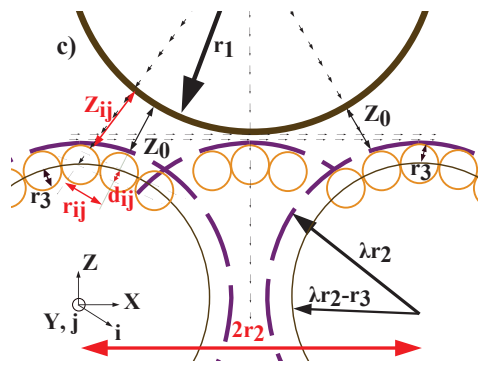

(c)

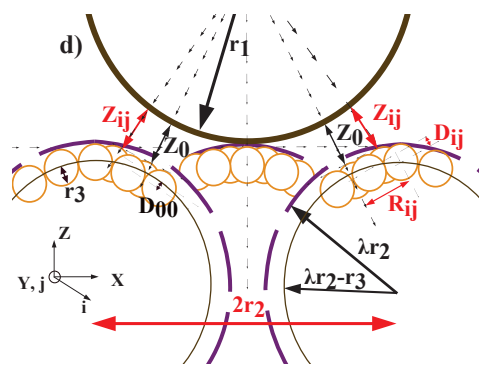

(d)
Fig. 6. Different interaction cases between the asperity $r_{3}$ on the PS sphere radius $\lambda r_{2}$ after the echting process and the probe $r_{1}$.

The new modeling force drawn in Figure 7 is :

$$
\begin{aligned}
& F_{\text {case } 1}=\sum_{i, j}^{\mathbb{Z}^{2}} \frac{A_{12} r_{1} r_{3}}{6 z_{i j \text { min }}^{2}\left(r_{1}+r_{3}\right)} \times \frac{r_{3}+z_{0}+r_{1}}{r_{3}+z_{i j}{ }_{m i n}+r_{1}} \\
& F_{\text {case } 2}=\sum_{i, j}^{\mathbb{Z}^{2}} \frac{A_{12} r_{1} r_{3}}{6 z_{i j \max }^{2}\left(r_{1}+r_{3}\right)} \times \frac{\sqrt{\left.\left(r_{3}+z_{i j} \max _{1}+r_{1}\right)^{2}-R_{i j}^{2}\right)}}{r_{3}+z_{i j_{\max }}+r_{1}}
\end{aligned}
$$

$$
\begin{aligned}
& F_{\text {case } 3}=\sum_{i, j}^{\mathbb{Z}^{2}} \frac{A_{12} r_{1} r_{3}}{6 z_{i j \text { min }}^{2}\left(r_{1}+r_{3}\right)} \times \frac{r_{3}+z_{0}+r_{1}}{r_{3}+z_{i j}+r_{1}} \\
& \times \frac{\sqrt{\left(\lambda r_{2}+z_{0}+r_{1}\right)^{2}-\left(4 / 3 \cdot r_{2}^{2}\right)}}{\lambda r_{2}+z_{0}+r_{1}} \\
& F_{\text {case } 4}=\sum_{i, j}^{\mathbb{Z}^{2}} \frac{A_{12} r_{1} r_{3}}{6 z_{i j}^{2}{ }_{\text {max }}\left(r_{1}+r_{3}\right)} \times \frac{\sqrt{\left.\left(r_{3}+z_{i j} j_{\text {max }}+r_{1}\right)^{2}-R_{i j}^{2}\right)}}{r_{3}+z_{i j_{\max }}+r_{1}} \\
& \times \frac{\sqrt{\left(\lambda r_{2}+z_{0}+r_{1}\right)^{2}-\left(4 / 3 \cdot r_{2}^{2}\right)}}{\lambda r_{2}+z_{0}+r_{1}}
\end{aligned}
$$

where:

$\begin{aligned} z_{i j_{\text {min }}} & =\sqrt{\left(r_{3}+z_{0}+r_{1}+d_{i j}\right)^{2}+r_{i j}^{2}}-r_{1}-r_{3} \\ z_{i j_{\text {max }}} & =\sqrt{R_{i j}^{2}+\left(D_{00}-d_{i j}-\sqrt{\left.\left(r_{3}+z_{0}+r_{1}\right)^{2}-\frac{4 r_{3}^{2}}{3}\right)^{2}}-r_{1}-r_{3}\right.}\end{aligned}$

$d_{i j}=\lambda r_{2}-\sqrt{\left(\lambda r_{2}-r_{3}\right)^{2}-r_{i j}^{2}}-r_{3}$

$D_{00}=\lambda r_{2}-\sqrt{\left(\lambda r_{2}-r_{3}\right)^{2}-\frac{4 r_{3}^{2}}{3}}-r_{3}$

$r_{i j}=2 r_{3} \sqrt{i^{2}+j^{2}-i j}$

$R_{i j}=2 r_{3} \sqrt{i^{2}+j^{2}-i j-j-\frac{1}{3}}$

where $\lambda$ is the PS radius sphere reduction coefficient due to the etching process.

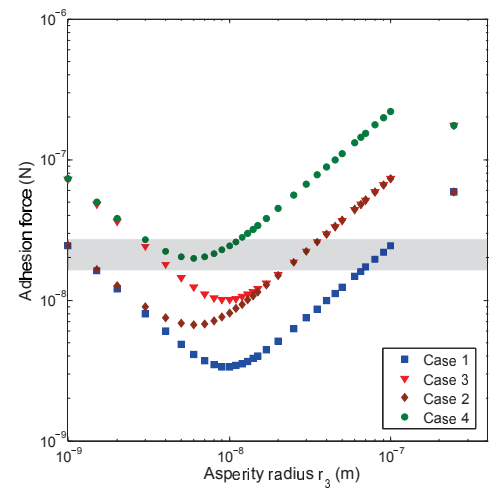

Fig. 7. Theoretical pull-off forces as a function of PS sphere roughness, $r_{3}$. The points are the theoretical values for the different cases and the grey aera is the experimental values.

In Figure 7, the first points on the right-hand of the figure are the pull-off forces without roughness (when $r_{3}=r_{2}$ ). The result deals with the determination of a minimum of the interaction force which represents an optimum of adhesion reduction in the applicative field of micromanipulation. In our experimental case, the optimum radius $r_{3}$ in order to minimize the adhesion is between $6 \mathrm{~nm}$ and $10 \mathrm{~nm}$. This optimum is reached for radius 10 times smaller than in a case of a structured plan (Figure 5) with a force also ten times smaller. This value depends (i) on the interaction case between the probe and the asperities; (ii) on the borosilicate sphere radius glued to the cantilever (nature and size) and (iii) on the initial radius $r_{2}$ of the sphere.

Particular asymptotic convergences can be observed. In the right-hand part of the figure, cases 2 and 3 are converging to the same values. In these cases, the probe is in interaction with only the sphere(s) at distance $z_{0}$. In the cases $1,2,3$ 
and 4 , the sphere(s) number in interaction with the probe are $1,3,3$ and 9 respectively. So the adhesion force is the same for the cases 2 and 3. In the left-hand part of the Figure 7 case 1 and 2 and case 3 and 4 are converging to the same values respectively. In these cases, the radius $r_{3}$ is lower than this optimum, and more and more spheres should be considered in the sum and the arrangement of the spheres is near to a plane surface, thus increasing the force. The spheres number on the sum is so important (more than 300 for $r_{3}=1 \mathrm{~nm}$ ) that the number of spheres $r_{3}$ in contact has no influence, and only the number of spheres $r_{2}$ should be taken into consideration. So the cases 1 and 3 is similar to the cases 2 and 4 respectively.

\section{Functionalised By Silane GRAFTing}

We have tested this approach using amine functions $\mathrm{NH}_{2}$ which can be protonated in $\mathrm{NH}_{3}^{+}$. The silane grafting used to placed amine functions on surface is described in [22], [23]. The measurements of interaction forces were performed in wet medium at different $\mathrm{pHs}$.

When only the grippers are functionalised, the interactions (attraction or repulsion) are controlled by the $\mathrm{pH}$ of the solution. At pH 5.5 the gripper and the object are attracted whereas at $\mathrm{pH}$ superior to 9 the both objects are pushed [23]. The force between a surface and a microsphere both functionalised by a grafting silane (APTES) layer are summarized in Figure 8.

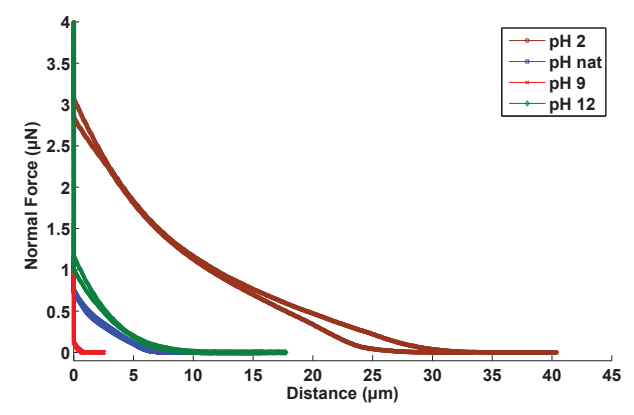

Fig. 8. Force-distance curves for APTES functionalised substrate in wet medium at different $\mathrm{pH}$ obtained with a sphere functionalised APTES (spring constant $0.3 \mathrm{~N} / \mathrm{m}$ ).

The forces measured in the liquid were always repulsive between the two surfaces. We did not detect any pull-off force. There is in fact no adhesion between both functionalised objects. A cantilever deformation was observed on an important distance (typically several micrometers) when the sphere is approaching from the surface. This large interaction distance typically comes from electrostatic interactions induced by the protonation. We note that the $\mathrm{pH}$ of the medium changes the value of the repulsive force between the cantilever and the surface but the behaviour stays always repulsive. For acidic and natural $\mathrm{pH}$, the repulsion can be explained by the positive charges of the aminosilane grafted on the surface. For basic $\mathrm{pH}$, the repulsion is induced by the negative charges of the silica substrate down to the functionalisation. Indeed, from $\mathrm{pH}$, the positive charges of the aminosilane are not sufficient to totally screening the negative charges of the silica. So the resulting negative charges are weak and the repulsion is less. Moreover, at $\mathrm{pH}$ 12, the aminosilane has any positive charges left and the repulsion is only induced by the negative charges on silica and borosilicate.

The interaction distance between the probe and the surface is simulated by Coulomb law between two surfaces whose surface charges are constant and determined from the maximum repulsive force for each $\mathrm{pH}$ [23]. A Finite Element Model (FEM) of the Coulomb force between a finite surface and a sphere with an identical surface charges $\sigma_{1}$ [23] has been simulated with the software COMSOL Multiphysics 3.5. Comparative results between experiments and simulated forces are presented in the Figure 9. It clearly shows that the model using Coulomb force between two charged surfaces is able to explain both the high long range of the interaction and the level of force.

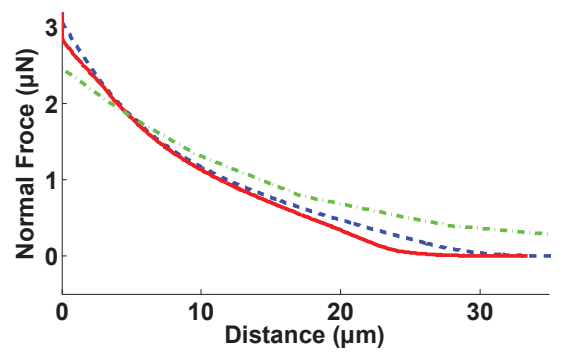

Fig. 9. Experimental and simulated force-distance curves for APTES functionalised substrate and cantilever at $\mathrm{pH}$ 2. The dash line (blue) is the experimental approach of the surface, the full line (red) is the experimental retract and the dash-dot line (green) is the simulated Coulomb force.

In micromanipulation, the repulsive charges between two objects are an interesting behavior in order to make easier the separation of two objects whatever the $\mathrm{pH}$ of the solution. These experiments were performed on the microassembly station developed in FEMTO-ST.

This repulsion, at $\mathrm{pH} 12$, was observed during the micromanipulation tasks. When we approach the functionalised Silicon Finger tip (SiFit) near the functionalised glass sphere, the repulsive force removes the glass to the SiFit (Figure 10). In the Figure 10, the sequence of the images is the sphere behavior when the SiFit approach the sphere. In spite of this repulsion force, it is possible to catch the ball with the gripper thanks to the two fingers. When the ball is caught, the sphere release is easy because of the repulsive force.

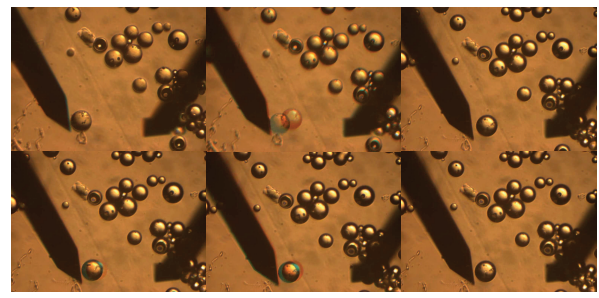

Fig. 10. Repulsion of the functionalised glass sphere when a functionalised Silicon Finger Tip (SiFit) approach to its. 


\section{DISCUSSION}

The techniques presented in this paper in order to decrease or control the adhesion present some advantages et disadvantages. In this part, we propose to compare the different techniques exposed previously versus their applications in micromanipulation. Two strategies (electropolymerisation and nanostructuration) can be used to reduce or cancel the adhesion in dry medium depending to the nature of the gripper and the size of the manipulated object. On conductive material and with manipulated objects bigger than $6 \mu \mathrm{m}$, the electropolymerisation must be preferred otherwise the structuration is better even through this method is more expensive and longer than the electropolymerisation. In order to control and cancel the adhesion in liquid media, the possibility is to graft the gripper or the manipulated object, or/and the both (gripper and object) depending to the gripper configuration: one or two fingers. For the first one, the manipulation (grasp and release) is control by the solution $\mathrm{pH}$, with only the one element functionalisation and for the second one the manipulation is improved if the object and the gripper are functionalised however the previous solution is again available. The disadvantage of the grafting is the no selectivity of the functionalised gripper and object parts. The chemical molecule is bound on all the oxyded surface.

\section{CONCLUSIONS AND FUTURE WORKS}

\section{A. Conclusions}

In this paper, we have studied interactions behavior between two functionalised surfaces and between functionalised and neutral surfaces. Different functionalisations as the grafting of aminosilane, the electrodeposition and the nanosphere deposition have been tested. All these techniques are a promising way in order to control, reduce or cancel the adhesion force during micromanipulation tasks. The microassembly could be improved by a judicious choice of the $\mathrm{pH}$ in liquid medium or of nanosphere structurations in function of the manipulated objects material size and nature in dry medium. If the conductivity of the grippers is an important point, it can be recovered by some intrinsic conducting polymers who decrease drastically the adhesion force.

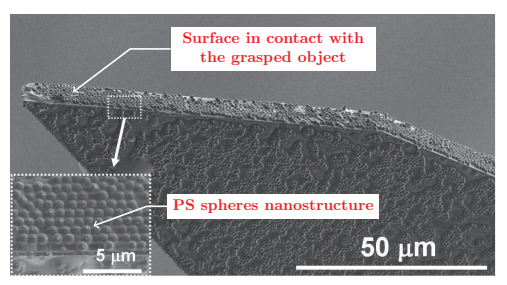

Fig. 11. Structured gripper by PS particles of $1 \mu \mathrm{m}$.

\section{B. Future Works}

This paper consists in a proof of concept of a new promising micromanipulation methods. The complete characterisation of these methods based on repeatability measurements as well as reliability determination has to be performed.
Future works will also focus on the implementation of these methods which are able to cancel adhesion perturbations on two-fingered microgrippers. Large blocking force required in microassembly will be thus possible. The first step for testing the repeatability of the micromanipulation task with a structured surface was overcame with the structuration of the finger tip (Figure 11).

\section{REFERENCES}

[1] G. Fantoni and M. Porta. A critical review of releasing strategies in microparts handling. In Micro-Assembly Technologies and Applications IFIP, volume 260, 2008.

[2] M. Gauthier and S. Régnier. Robotic micro-assembly. 2010.

[3] R. Bogue. Assembly of 3d micro-components: a review of recent research. Assembly Automation, 31(4):309-314, 2011

[4] Y. Rollot, S. Régnier, and J-C. Guinot. Dynamical model for the micromanipulation by adhesion: experimental validations for determined conditions. Journal of Micromechatronics, 1(4):273-297, 2001.

[5] W. Driesen, T. Varidel, S. Régnier, and J.M. Breguet. Micromanipulation by adhesion with two collaborating mobile micro robots. Journal of Micromechanics and Microengineering, 15:259-267, 2005.

[6] M. Gauthier, E. Gibeau, and D. Hériban. Submerged robotic micromanipulation and dielectrophoretic micro-object release. In Proc. of the IEEE ICARCV 2006 conference, Singapour, 2006. IEEE.

[7] D. Hériban, et al. Automatic pick-and-place of 40 microns objects using a robotic platform. In proc. of Proceedings of the 9th euspen International Conference, San Sebastian, Spain, June 2009.

[8] Q. Zhou V. Sariola and H. N. Koivo. Hybrid microhandling: a unified view of robotic handling and self-assembly. Journal of Micro - Nano Mechatronics, 4:5-16, 2008.

[9] F. Arai. Microrobots in spotlight for evolution of biomedicine. In IEEE Int. Conf. on MEMS, 2012.

[10] K. Onda and F. Arai. Multi-beam bilateral teleoperation of holographic optical tweezers. Optics Express, 20(4):3633-3641, 2012.

[11] M. Kharboutly, M. Gauthier, and N. Chaillet. Predictive control of a micro bead's trajectory in a dielectrophoresis-based device. In IEEE/RSJ IROS, Taipei, Taiwan, oct. 2010.

[12] M. Bechelany, P. Brodard, J. Elias, L. Philippe, and J. Michler Simple synthetic route for sers active gold nanoparticles substrate with controlled shape and organization. Langmuir, 26:14364-14371, 2010.

[13] M. Bechelany, E. Berodier, X. Maeder, S. Schmitt, J. Michler, and L. Philippe. New silicon architectures by gold-assisted chemical etching. ACS Appl. Mater. Interfaces, 3:3866, 2011.

[14] S. Lupu, B. Lakard, J. Hihn, J. Dejeu, P. Rougeot, and S. Lallemand. Morphological characterisation and analytical application of poly(3,4-ethylenedioxythiophene)-prussian blue composite films electrodeposited in situ on platinium electrode chips. Thin Solid Films, 519:7754-7762, 2011.

[15] S. Lupu, B. Lakard, J. Hihn, and J. Dejeu. Novel in situ electrochemical deposition of platinum nanoparticles by sinusoidal voltages on conducting polymer films. Synthetic Metals, 162:193-198, 2012.

[16] J. Dejeu, et al. Morphological and adhesive properties of polypyrrole films synthesized by (sono)electrochemistry. Synthetic Metals, 160:2540-2545, 2010.

[17] J. Dejeu, et al. Control of the adhesion force with the polymers electrodeposit. submitted in Thin Solid Films.

[18] F. Sekli-Belaidi, P. Temple-Boyer, and P. Gros. Voltammetric microsensor using pedot-modified gold electrode for the simultaneous assay of ascorbic and uric acids. Journal of Electroanalytical Chemistry, 647:159-168, 2010.

[19] Y. Xiao, X. Cui, and D.C. Martin. Electrochemical polymerization and properties of pedot/s-edot on neural microelectrode arrays. Journal of Electroanalytical Chemistry, 573:43-48, 2004.

[20] J. Dejeu, et al. Adhesion control for micro- and nano-manipulation. ACS Nano, 5:4648-4657, 2011.

[21] Y. P. Zhao, J. T. Drotar, G. C. Wang, and T. M. Lu. Physical Review Letters, 82:4882-4885, 1999.

[22] S.R. Wasserman, Y.-T. Tao, and G.M. Whitesides. Structure and reactivity of alkylsiloxane monolayers formed by reaction of alkyltrichlorosilanes on silicon substrates. Langmuir, 5:1074-1087, 1989.

[23] J. Dejeu, et al. Adhesions forces controlled by chemical self-assembly and ph, application to robotic microhandling. ACS Applied Materials \& Interfaces, 1:1966-1973, 2009. 